Gastric cancer is one of the most frequent neoplasms. Although the incidence of gastric cancer worldwide has declined, there is still high mortality. Treatment of inoperable disease is under evaluation in clinical trials. In palliative treatment chemotherapy containing cisplatin and 5-fluorouracil is the most widely used.

In the past years progress in tumour biology has advanced greatly and has led to development of new molecules aimed at targets important for cancer expansion. There are several randomized trials under targeted therapies for gastric cancer patients. One of them led to approval of trastuzumab.

In the current paper the authors illustrate new possibilities in systemic treatment with particular attention to targeted therapy and personalization in medicine.

Key words: gastric cancer, chemotherapy, targeted therapy, personalization in medicine.

\section{Chemotherapy for gastric cancer patients - time for personalization in medicine?}

\author{
Elżbieta Nowara, Agnieszka Boratyn-Nowicka, Anna Polakiewicz-Gilowska, \\ Anna Drosik, Magdalena Kustra, Joanna Huszno
}

Clinical and Experimental Oncology Department, Cancer Centre - Institute of Oncology - MSC Gliwice Branch, Poland

\section{Introduction}

Gastric cancer (GC) is one of the most common cancer types. The majority of gastric tumours (90-95\%) are adenocarcinomas. Other types include lymphomas (2/3) and sarcomas (1/4).

Both morbidity and mortality due to gastric cancer have recently shown a downward trend in most countries worldwide. Reduced morbidity has also been observed in Poland, though as yet without a significant reduction in mortality rates. According to National Cancer Registry data, a total of 5103 new cases were diagnosed in Poland in 2008. Also, a total of 5674 deaths from this cancer type were reported in the same year [1].

Poor prognosis is mainly due to late diagnosis. Median survival of patients with inoperative or metastatic gastric cancer (IMGC) does not exceed 10 months, while the maximum two-year survival rate is 10\% [2]. Patients in whom surgery is not possible due to advanced cancer stage usually require palliative treatment in the form of chemotherapy. Although a dozen or so different chemotherapy regimens are used in patients with inoperable cancer to improve their quality of life, no significant extension of overall survival has been achieved so far. Likewise, no standard of care has been firmly established yet. The most commonly used cytostatics are cisplatin (DDP) and 5-fluorouracil (5FU) in various combinations.

The article outlines the evolution of systemic treatment of gastric cancer throughout the last several years and discusses results of the most recent studies into the so-called personalization in medicine.

\section{Chemotherapy versus best supportive care}

Even back in the 1990s, there was no consensus about the benefits of systemic treatment in IMGC patients. At that time, results of several phase III trials comparing chemotherapy with best supportive care (BSC) were published. Some of the trials were stopped prematurely because early evidence was obtained both in terms of significant extension of progression-free survival (PFS) and overall survival (OS) among patients receiving chemotherapy compared to BSC, $p<0.05[3,4]$. Furthermore, the quality of life of chemotherapy patients was found to be better than BSC patients [5].

\section{Single-drug chemotherapy versus combination chemotherapy}

Also, more than ten trials were conducted to compare single-drug with multiple-drug chemotherapy regimens. Seven of the trials were meta-analyzed. Overall, the trials involved 1472 patients [2]. Most investigators used 5FU in the monochemotherapy arm. The same agent was also used in multiple-drug regimens [5-7]. Authors of the meta-analysis stress that toxicity was insignificantly higher in patients receiving multiple-drug chemotherapy than sin- 
gle-drug chemotherapy. Response rates were higher in the multiple-drug chemotherapy arm, $p<0.00001$. Also, progression-free survival ( $p=0.015$ ) and overall survival $(p=0.00003)$ times were shown to be significantly longer in patients treated with multiple-drug regimens.

\section{Comparison of various multiple-drug regimens}

IMGC patients have also been studied in about a dozen phase III clinical trials seeking to establish the standard regimen of palliative chemotherapy. The most commonly used drug combinations included 5FU, DDP, methotrexate (MTX), adriamycin (ADM), etoposide (VP-16) and mitomycin C. Wagner's metaanalysis investigating two and three-drug regimens demonstrated longer overall survival in patients receiving threedrug combination chemotherapy. Drug regimens including anthracyclines were also shown to be superior in terms of efficacy to anthracycline-free regimens [8]. Cisplatin-treated patients were found to have a longer survival than patients receiving cisplatin-free chemotherapy $[9,10]$. The quality of life of patients treated with DDP-containing regimens was better than patients treated without cisplatin. Some authors recognize the ECF scheme (epirubicin, DDP, 5FU) as the standard of care for IMGC patients [21]. Others claim that no three-drug combination chemotherapy extends the overall survival compared to the two-drug regimen DDP plus 5FU. Finally, it was observed that three-drug regimens might extend progression-free survival and increase the treatment response rate $[8,12,13]$.

Recent years have seen the publication of results of several phase III trials investigating new drugs in gastric cancer patients, including fluoropyrimidines, irinotecan, taxanes and oxaliplatin.

The REAL-2 trial demonstrated that DDP substitution with oxaliplatin eliminated the requirement for lengthy hydration and reduced the risk of complications (renal dysfunction, hearing disorders, vomiting). Capecitabine administered instead of prolonged continuous infusions with 5FU was found to improve the quality of life of patients without compromising therapeutic efficacy [13]. Taxanes added to DDP and 5FU (DCF) regimens increase response rates, and extend progressionfree survival and overall survival [14]. Due to considerable toxicity (neutropenic fever developing in 30\% of patients), DCF was not adopted as the standard of care in IMGC patients.

Irinotecan-based regimens have also been assessed in phase III trials. Patients treated with irinotecan had longer overall survival than patients receiving irinotecan-free chemotherapy. Trials comparing the standard regimen (i.e. DDP in combination with 5FU [PF]) demonstrated an insignificantly lower occurrence rate of lethal complications in patients treated with chemotherapy regimens containing irinotecan $[15,16]$.

Today, PF (DDP, 5FU) remains the most commonly used palliative chemotherapy regimen in IMGC patients worldwide. Response rates do not usually exceed $20-30 \%$, while oneyear survival rates reach $30 \%$, with a median survival time of approximately 8 months [13].

\section{Molecular targeted therapy}

New molecularly targeted drugs, commonly referred to as targeted therapy, are increasingly used in clinical practice to treat patients with malignancies. For example, trastuzumab has been approved for patients with HER2-overexpressing breast cancer, while bevacizumab, cetuximab and panitumumab have been approved for colorectal cancer.

The epidermal growth factor receptor (EGFR) pathway is a commonly known pathway which plays an important role in oncogenesis. EGFR overexpression is often seen in excised specimens of malignant gastrointestinal tumours. In GC patients, HER2 overexpression is more common in the intestinal type, tumours located proximally or in the gastroesophageal junction, higher-stage cancer, spreading to the lymph nodes and at the stage of liver metastases; it is associated with more rapid disease progression and shorter survival $[17,18]$.

Results of a phase III trial on the addition of trastuzumab to PF chemotherapy in HER2 overexpressing patients (ToGa) were presented during the 2009 ASCO Meeting. Overall survival was found to be significantly longer among patients in the trastuzumab arm (13.5 vs. 11 months), $p=0.0048$ [19]. On that basis, trastuzumab was approved for the therapy of patients with metastatic gastric cancer and HER2 overexpression.

Results of the ToGa trial demonstrating the efficacy of targeted therapy in a selected group of patients created a foundation for further studies into EGFR inhibitors in GC therapy. Also, by analogy to breast cancer treatment, lapatinib is currently being investigated as second-line therapy in gastric cancer patients who have previously failed trastuzumab treatment.

K-RAS or B-RAF mutations are rarely observed in excised specimens of malignant gastric tumours. The finding forms a basis for attempts to use cetuximab or bevacizumab for GC treatment. For the time being, the only reports of efficacy of antiangiogenic therapy in IMGC patients come from nonrandomized phase II trials. In one of them, bevacizumab was combined with irinotecan and DDP for first-line therapy. The response rates were $65 \%$, while progression-free survival was 8.3 months and overall survival was 12.3 months. At the same time, however, bevacizumab was associated with frequent severe complications, mostly thromboembolic incidents and gastrointestinal perforations [20, 21]. In another trial, bevacizumab was combined with the DCF regimen. Similar results were noted [22]. Results of the AVAGAST phase III trial designed to evaluate the efficacy of bevacizumab combined with capecitabine and DDP, compared to chemotherapy alone, are currently being awaited. MAGIC-2, another trial which is now in progress, seeks to evaluate the efficacy of bevacizumab for neoadjuvant treatment. Yet another trial, EXPAND, is designed to clarify the role of cetuximab combined with DDP and capecitabine in first-line treatment of IMGC patients.

Also, results are yet to be announced for trials investigating combinations of chemotherapy with other angiogenesis inhibitors (sunitinib, sorafenib, cediranib or axitinib), other EGFR inhibitors (panitumumab, matuzumab) and inhibitors of intracellular tyrosine kinase domain (gefitinib, erlotinib, lapatinib, canertinib).

Researchers expect that new molecules will improve efficacy levels and reduce chemotherapy-induced toxicity in patients suffering from disseminated gastric cancer. 
However, with the exception of trastuzumab, no targeted drug has as yet been approved for the therapy of GC patients.

As is commonly known, GC is disseminated both by blood and lymph circulation, and into the peritoneum. Some authors point to an increased risk of digestive obstruction during irinotecan treatment in patients with GC metastasizing to the peritoneum. Japanese studies have shown the survival rates of patients with peritoneal metastases treated with irinotecan-containing regimens to be significantly shorter compared to patients treated without irinotecan. On the other hand, the survival rates of patients receiving the same therapy, though with different metastatic sites, were found to be longer [15, 23]. S-1 (tegafur, gimeracil, oteracil), which is widely used in Asia, is thought to be more toxic in Caucasian than Asian populations. Two trials (FLAGS and S.C.-101) demonstrated longer OS survival in patients treated with the S-1 regimen combined with DDP in the Chinese and Japanese populations compared to the Caucasian population, in which the drugs were also found to be more toxic [23-25].

\section{Second-line therapy and beyond}

Approximately half of GC patients fail their first-line therapy. The majority of other patients experience tumour progression during the course of the disease $[24,26]$. It is not clear whether second-line systemic treatment, followed by thirdline and beyond, has an effect on the survival of GS patients. It seems, however, that GC patients may benefit from this type of management similarly to patients suffering from colorectal cancer. A phase II trial assessing the efficacy of irinotecan combined with capecitabine in patients who have failed their DDP $+5 \mathrm{FU}$ therapy has shown that with reduced drug doses the regimen is safe and produces high response rates [27].

The growing number of new active and easily accessible drugs gives grounds to believe that patients may benefit from further therapy, even though biological differences between gastric and colorectal cancers are indisputable.

Regardless of the number and quality of available drugs, however, it should be remembered that advanced gastric cancer is associated with extremely poor prognosis. Therefore, the overriding goal of therapy should always be to improve the quality of patients' life. Palliative radiotherapy is another effective method ensuring analgesic and anti-haemorrhagic effects [28].

\section{Summary}

Since new therapies, opening up new treatment possibilities, are becoming more easily accessible, GC patients and their families can hope for more effective and safer treatment. Expanding knowledge of GC biology makes it possible to individualize treatment to the patient, not only to cancer type. For example, chemotherapy can be combined with trastuzumab in GC patients with HER-2 overexpression. Some authors claim that patients with peritoneal metastases should not be treated with irinotecan because of elevated risk of digestive obstruction. In Caucasian patients S-1 is more toxic and less effective than in the Asian population.

The outcomes of numerous studies into new targeted drugs for GC therapy are currently awaited.
Bad prognosis should, however, always be borne in mind during IMGC therapy [33]. Patients who are in poor general condition (ZUBROD score $\geq 2$ ) should not be deemed eligible for systemic treatment. Patients with a ZUBROD score $<2$, depending on their comorbidities and therapeutic possibilities of the medical centre, can receive systemic or supportive therapy, or be enrolled in clinical trials (NCCN v.2010 Gastric Cancer, 34). Such therapeutic management may be opening the door for personalized medicine in gastric cancer.

\section{References}

1. http://epid.coi.waw.pl/krn/

2. Wagner AD, Grothe W, Haerting J, Kleber G, Grothey A, Fleig WE. Chemotherapy in advanced gastric cancer: a systematic review and meta-analysis based on aggregate data. J Clin Oncol 2006; 24: 2903-9.

3. Murad AM, Santiago FF, Petroianu A, Rocha PR, Rodrigues MA, Rausch M. Modified therapy with 5-fluorouracil, doxorubicin, and methotrexate in advanced gastric cancer. Cancer 1993; 72: 37-41.

4. Pyrhönen S, Kuitunen T, Nyandoto P, Kouri M. Randomised comparison of fluorouracil, epidoxorubicin and methotrexate (FEMTX) plus supportive care with supportive care alone in patients with nonresectable gastric cancer. Br J Cancer 1995; 7: 587-91.

5. Glimelius B, Ekström K, Hoffman K, et al. Randomized comparison between chemotherapy plus best supportive care with best supportive care in advanced gastric cancer. Ann Oncol 1997; 8: 163-8.

6. Cullinan SA, Moertel CG, Wieand HS, O'Connell MJ, Poon MA, Krook JE, Mailliard JA, Tschetter LK. Controlled evaluation of three drug combination regimens versus fluorouracil alone for the therapy of advanced gastric cancer. North Central Cancer Treatment Group. J Clin Oncol 1994; 12: 412-6.

7. De Lisi V, Cocconi G, Angelini F, et al. The combination of cisplatin, doxorubicin, and mitomycin (PAM) compared with the FAM regimen in treating advanced gastric carcinoma. A phase II randomized trial of the Italian Oncology Group for Clinical Research. Cancer 1996; 77: 245-50.

8. Kim NK, Park YS, Heo DS, et al. A phase III randomized study of 5-fluorouracil and cisplatin versus 5 -fluorouracil, doxorubicin, and mitomycin C versus 5-fluorouracil alone in the treatment of advanced gastric cancer. Cancer 1993; 71: 3813-8.

9. Roth A, Kolaric K, Zupanc D, Oresic V, Roth A, Ebling Z. High doses of 5-fluorouracil and epirubicin with or without cisplatin in advanced gastric cancer: a randomized study. Tumori 1999; 85: 234-8.

10. Cocconi G, Carlini P, Gamboni A, et al. talian Oncology Group for Clinical Research. Cisplatin, epirubicin, leucovorin and 5-fluorouracil (PELF) is more active than 5-fluorouracil, doxorubicin and methotrexate (FAMTX) in advanced gastric carcinoma. Ann Oncol 2003; 14: 1258-63.

11. Webb A, Cunningham D, Scarffe JH, et al. Randomized trial comparing epirubicin, cisplatin, and fluorouracil versus fluorouracil, doxorubicin, and methotrexate in advanced esophagogastric cancer. J Clin Oncol 1997; 15: 261-7.

12. Vanhoefer $U$, Rougier $P$, Wilke $H$. et al. Final results of a randomized phase III trial of sequential high-dose methotrexate, fluorouracil, and doxorubicin versus etoposide, leucovorin, and fluorouracil versus infusional fluorouracil and cisplatin in advanced gastric cancer: A trial of the European Organization for Research and Treatment of Cancer Gastrointestinal Tract Cancer Cooperative Group. J Clin Oncol 2000; 18: 2648-57.

13. Cunningham D, Starling N, Rao S, et al. Capecitabine and oxaliplatin for advanced esophagogastric cancer. N Engl J Med 2008; 358: 36-46.

14. Van Cutsem E, Moiseyenko VM, Tjulandin S, et al. Phase III study of docetaxel and cisplatin plus fluorouracil compared with cisplatin and fluorouracil as first-line therapy for advanced gastric cancer: a report of the V325 Study Group. J Clin Oncol 2006; 24: 4991-7.

15. Boku N, Yamamoto S, Fukuda H, et al. Gastrointestinal Oncology Study Group of the Japan Clinical Oncology Group. Fluorouracil versus com- 
bination of irinotecan plus cisplatin versus S-1 in metastatic gastric cancer: a randomised phase 3 study. Lancet Oncol 2009; 10: 1063-9.

16. Dank M, Zaluski J, Barone C, et al. Randomized phase III study comparing irinotecan combined with 5 -fluorouracil and folinic acid to cisplatin combined with 5-fluorouracil in chemotherapy naive patients with advanced adenocarcinoma of the stomach or esophagogastric junction. Ann Oncol 2008; 19: 1450-7.

17. Kitagawa Y, Ueda M, Ando N, Ozawa S, Shimizu N, Kitajima M. Further evidence for prognostic significance of epidermal growth factor receptor gene amplification in patients with esophageal squamous cell carcinoma. Clin Cancer Res 1996; 2: 909-14.

18. Park DI, Yun JW, Park JH, et al. HER-2/neu amplification is an independent prognostic factor in gastric cancer. Dig Dis Sci 2006; 51: 1371-9.

19. Bang YJ, Van Cutsem E, Feyereislova A, et al. ToGA Trial Investiga tors. Trastuzumab in combination with chemotherapy versus chemotherapy alone for treatment of HER2-positive advanced gastric or gastro-oesophageal junction cancer (ToGA): a phase 3, openlabel, randomised controlled trial. Lancet 2010; 376: 687-97.

20. Shah MA, Jhawer M, Ilson DH, Lefkowitz RA, Robinson E, Capanu M, Kelsen DP. Phase II Study of modified docetaxel, cisplatin, and fluorouracil with bevacizumab in patients with metastatic gastroesophageal adenocarcinoma. J Clin Oncol 2011; 29: 868-74.

21. Shah MA, Ramanathan RK, Ilson DH, et al. Multicenter phase II study of irinotecan, cisplatin, and bevacizumab in patients with metastatic gastric or gastroesophageal junction adenocarcinoma. J Clin Oncol 2006; 24: 5201-6.

22. Jhawer M. Phase II study of modified docetaxel, cisplatin, fluorouraci (mDCF), and bevacizumab (BEV) in patients with metastatic gastroesophageal (GE) adenocarcinoma (abstract \#10). American Society of Clinical Oncology GI Cancer Symposium. 2009.

23. Narahara H, lishi H, Imamura H, et al. Randomized phase III study of irinotecan plus S-1 (IRIS) versus S-1 alone as first-line treatment for advanced gastric cancer (GC0301/TOP-002). Gastric Cancer 2011; 14: $72-80$

24. Okines A, Verheij M, Allum W, Cunningham D, Cervantes A; ESMO Guidelines Working Group. Gastric cancer: ESMO Clinical Practice Guidelines for diagnosis, treatment and follow-up. Ann Oncol 2010; 21 Suppl 5: v50-4.

25. Boku N. Perspectives for personalization in chemotherapy of advanced gastric cancer. Discov Med 2010; 9: 84-9.

26. Javle M, Hsueh CT. Recent advances in gastrointestinal oncology updates and insights from the 2009 annual meeting of the American society of clinical oncology. J Hematol Oncol 2010, 3: 11.

27. Sun Q, Hang M, Xu W, Mao W, Hang X, Li M, Zhang J. Irinotecan plus capecitabine as a second-line treatment after failure of 5 -fluorouracil and platinum in patients with advanced gastric cancer. Jpn J Clin Oncol 2009; 39: 791-6.

28. Asakura $\mathrm{H}$, Hashimoto $\mathrm{T}$, Harada $\mathrm{H}$, et al. Palliative radiotherapy for bleeding from advanced gastric cancer: is a schedule of $30 \mathrm{~Gy}$ in 10 fractions adequate? J Cancer Res Clin Oncol 2011; 137: 125-30.

\section{Address for correspondence}

\section{Elżbieta Nowara}

Klinika Onkologii Klinicznej i Doświadczalnej

Centrum Onkologii - Instytut im. Marii Skłodowskiej-Curie

Oddział w Gliwicach

Wybrzeże Armii Krajowej 15

44-100 Gliwice

tel. +4832 2788717

e-mail: enowara@io.gliwice.pl

Submitted: $\quad 14.02 .2011$

Accepted: $\quad$ 18.01.2012 